\title{
Autophagy in human articular chondrocytes is cytoprotective following glucocorticoid stimulation
}

\author{
NING LIU, WENBO WANG, ZHE ZHAO, TAO ZHANG and YUWEN SONG \\ Department of Orthopaedic Surgery, The First Affiliated Hospital of Harbin Medical University, \\ Harbin, Heilongjiang 150001, P.R. China
}

Received August 13, 2013; Accepted March 7, 2014

DOI: $10.3892 / \mathrm{mmr} .2014 .2102$

\begin{abstract}
For the past 60 years, glucocorticosteroid (GC) drugs, including prednisone and dexamethasone (Dex), have been used for the treatment of early stage osteoarthritis (OA). However, multiple administration of GCs may destroy the articular cartilage. It has been previously reported that GC treatment may also lead to the initiation of autophagy, which is an essential mechanism for cell homeostasis and survival. Rapamycin (Rapa), an inhibitor of the mammalian target of Rapamycin, may cause a degeneration-associated pathology in organs and induce autophagy in a variety of cell types, which has been applied in the treatment of experimental OA. A previous study by our group observed that GC apparently increases the apoptosis of chondrocytes, resulting in the inhibition of extracellular matrix synthesis. Therefore, the present study aimed to further examine the effects of autophagy in chondrocytes under GC treatment and to verify the molecular mechanisms involved in the cytoprotective role of Rapa. Short-term GC treatment did not significantly inhibit chondrocyte viability, while cell autophagy was increased. In addition, upregulation of autophagy by Rapa prevented the expression of apoptosis-associated genes and improved cell activity. In conclusion, the present study revealed that increased autophagy is an adaptive response to protect chondrocytes from short-term GC exposure, whereas prolonged GC treatment decreases autophagy and increases apoptosis in vitro. Upregulation of autophagy by Rapa may protect chondrocytes against the adverse effect induced by GC.
\end{abstract}

\section{Introduction}

Osteoarthritis (OA) is the most prevalent type of joint disease in adults and is characterized by chronic pain and

Correspondence to: Professor Wenbo Wang, Department of Orthopaedic Surgery, The First Affiliated Hospital of Harbin Medical University, 23 You Zheng Street, Harbin, Heilongjiang 150001, P.R. China

E-mail:wangwwbo@126.com

Key words: chondrocytes, dexamethasone, glucocorticoid, autophagy, apoptosis degradation of articular cartilage $(1,2)$. Treatments for early stage OA initially focus on reducing pain and inflammation, and preventing the cartilage from further mechanical injury. Glucocorticosteroids (GCs) are intra-aritcular therapeutics first used in for anti-inflammatory treatment of rheumatoid arthritis in 1951 and are effective in pain relief and functional improvement (3-6). However, long-term and/or high-dose treatment with GCs may be harmful for articular cartilage metabolism and may induce apoptosis in chondrocytes by destroying the endoplasmic reticulum and mitochondria $(7,8)$. In cartilage, chondrocytes are the sole resident cells that are involved in the synthesis of the extracellular matrix (ECM). Cartilage homeostasis requires sufficient ECM composition to exert its biomechanical functions. Therefore, inhibition of the growth of chondrocytes may reduce cartilage formation and possibly weaken the matrix structure.

Autophagy is characterized by the formation of an autophagosome. As the essential cellular homeostasis mechanism, the autophagosome may recycle the damaged and dysfunctional organelles and molecules $(9,10)$. Therefore, autophagy is considered a protective process for cell survival under metabolic stress and adverse stimulus (11). Thus, impairment of autophagy may induce a variety of human diseases. Due to the relatively low rate of proliferation of chondrocytes, autophagy appears to be important in maintaining cell survival and biosynthetic function $(12,13)$. However, whether the interaction between autophagy and apoptosis is involved in the effect of GC on normal human chondrocytes remains to be elucidated.

Rapamycin (Rapa) is a lipophilic macrolide antibiotic, which triggers autophagic functions by inhibiting the mammalian target of rapamycin (mTOR) (14). mTOR, a nutrient-sensing kinase which acts as a controller of ribosomal biogenesis and protein synthesis, may directly suppress cell autophagy (15). In addition, imbalances of this kinase are involved in metabolic diseases (16). Based on the potential of inducing autophagy, Rapa has been suggested to protect against certain degenerative conditions (17-19).

Recently, a study by our group demonstrated that short-term GC treatment did not suppress chondrocyte growth significantly, whereas long-term exposure to GCs induced apoptosis (20). Thus, the aim of the present study was to determine whether autophagy is one of the survival pathways through which chondrocytes respond to short-term GC treatment and whether pharmacological enhancement of autophagy 
by Rapa may serve as a protective mechanism against the adverse effects of long-term GC exposure.

\section{Materials and methods}

Chemicals and reagents. Dexamethasone (Dex), Rapa, monodansylcadaverine (MDC), Hoechst 33342 and dimethylsulfoxide (DMSO) were purchased from Sigma-Aldrich (St. Louis, MO, USA). A CCK-8 Cell Counting Kit was obtained from Beyotime Laboratories (Beyotime Institute of Biotechnology, Haimen, China). Annexin V-fluorescein isothiocyanate (FITC) and propidium iodide (PI) were purchased from BaoSai Biotechnology Co., Ltd. (Beijing, China). Antibodies against microtubule-associated protein light chain 3 (LC3), beclin-1, caspase-9, poly (adenosine diphosphate-ribose) polymerase (PARP), collagenase II, aggrecan and $\beta$-actin were purchased from Santa Cruz Biotechnology, Inc. (Santa Cruz, CA, USA).

Human chondrocyte isolation and culture. Human articular cartilage tissue samples from the knee joint were obtained from 10 donors (average age, 50.2 years) undergoing surgery for tibial plateau fracture or total knee arthroplasty. These surgeries were approved by the ethics committee of The First Affiliated Hospital of Harbin Medical University (Harbin, China). The non weight-bearing area of the cartilage, without any abnormalities, was harvested and washed in sterilized saline. Next, the tissue was sliced into smaller sections of $1 \mathrm{~mm}^{3}$ and digested with trypsin and collagenase II for $5 \mathrm{~h}$ at $37^{\circ} \mathrm{C}$. The mixture was filtered, centrifuged $(0.2 \times \mathrm{g})$ and washed in phosphate-buffered saline (PBS) at least three times for cell purification. Finally, the cells were transferred into a culture flask and incubated at $37^{\circ} \mathrm{C}$ in a $5 \% \mathrm{CO}_{2}$ incubator with $10 \%$ fetal bovine serum in Dulbecco's modified Eagle medium (DMEM) (Gibco-BRL, Carlsbad, CA, USA). Following the above procedure, the second-passage chondrocytes were used for further experiments.

Cell viability assay. Chondrocytes $\left(8 \times 10^{3}\right)$ were seeded into 96 -well plates and cultured for $24 \mathrm{~h}$. Based on the previous results, the cells were treated with $100 \mu \mathrm{M} / 1$ Dex in culture medium for the following times: $6,12,24,48$ or $72 \mathrm{~h}$. Cells were treated with DMSO only as the untreated control (control group), and one group was pretreated with $10 \mu \mathrm{M} / 1$ Rapa for $2 \mathrm{~h}$. At each time-point, $10 \mu \mathrm{l}$ CCK- 8 kit solution was added to each well and plates were incubated at $37^{\circ} \mathrm{C}$ for $\sim 1 \mathrm{~h}$. Next, a microplate reader (Thermo Fisher Scientific Inc., Walthom, MA, USA) was used to measure the optical density (OD) at $450 \mathrm{~nm}$. The experiment was repeated at least five times. The survival rate of cells $(\%)=($ experimental group OD value - blank group OD value)/(control group OD value - blank group OD value), and the inhibition rate of cell proliferation (\%) equals to $100 \%$ minus the survival rate.

Flow cytometric assessment of apoptosis. Following the respective treatment as above, the cells were washed with ice-cold PBS, harvested and counted. The cells $\left(1 \times 10^{5}\right)$ were suspended in $500 \mu \mathrm{l}$ binding buffer containing Hepes/ $\mathrm{NaOH}$ (pH 7.4), $\mathrm{NaCl}$ and $\mathrm{CaCl}_{2}$ (BaoSai Biotechnology Co., Ltd.) and incubated with $10 \mu \mathrm{l}$ Annexin V-FITC and $5 \mu \mathrm{l}$ PI for
$20 \mathrm{~min}$. The rate of apoptosis (\%) was measured by a flow cytometer (Epics Altra II; Beckman Coulter Inc., Brea, CA, USA). In addition, for Hoechst staining, the cells were seeded into 6-well plates and cultured for $24 \mathrm{~h}$. After treatment with or without $100 \mu \mathrm{M}$ of Dex for $72 \mathrm{~h}$ post-treatment, the cells were washed in cold PBS twice and were labeled with Hoechst 33258 staining solution (Beyotime Institute of Biotechnology), and maintained at $37^{\circ} \mathrm{C}$ in the dark for $20 \mathrm{~min}$. The cells were then observed and imaged by fluorescence microscopy (Leica Microsystems, Wetzlar, Germany), with excitation at $350 \mathrm{~nm}$ and emission at $460 \mathrm{~nm}$.

MDC assay. Chondrocytes were seeded on sterile coverslips in tissue culture plates. Following treatment with Dex and/or Rapa for the indicated times, the cells were incubated with $\operatorname{MDC}(0.1 \mathrm{mM})$ for $30 \mathrm{~min}$ at $37^{\circ} \mathrm{C}$. Following incubation, cells were washed three times with PBS and immediately visualized by fluorescence microscopy (Leica Microsystems). Excitation wavelengths were 360-380 nm and Olympus DP version software (Olympus Corporation, Tokyo, Japan) was used.

Transmission electron microscopy (TEM) analysis. Chondrocytes were treated with Dex and/or for designated times as described for the MDC assay. Cells were then washed with $0.1 \mathrm{M}$ cacodylate buffer $(\mathrm{pH} 7.4)$ and fixed with $2 \%$ glutaraldehyde in PBS for $24 \mathrm{~h}$ at $4^{\circ} \mathrm{C}$. Samples were then processed following the standard procedure (21). A Zeiss transmission electron microscope (Carl Zeiss, Thornwood, NY, USA) was used to examine the section samples.

Western blot analysis. Chondrocytes $\left(2 \times 10^{6}\right)$ were pretreated with or without Rapa $(10 \mu \mathrm{M} / \mathrm{l}) 2 \mathrm{~h}$ prior to Dex treatment. The cells were collected and western blot analysis was performed as previously described (19). Briefly, the cells were washed with ice-cold PBS and sonicated in radioimmunoprecipitation assay buffer and homogenized and cellular debris was removed by centrifugation $(0.2 \mathrm{x}$ g). The protein concentration was determined using the Bio-Rad DC protein assay (Bio-Rad Laboratories, Hercules, CA, USA). The protein lysates were separated by 12 or $15 \%$ SDS-PAGE and transferred to polyvinylidene difluoride membranes. The membranes were blocked with $5 \%$ bovine serum albumin (Beyotime Institute of Biotechnology) and then incubated with LC3 and Beclin-1 primary antibodies (1:500), Cleaved caspase-9 and PARP primary antibodies $(1: 1,000)$ overnight at $4^{\circ} \mathrm{C}$ and subsequently with horseradish peroxidase-conjugated anti-rabbit $(1: 1,500)$ or anti-mouse $(1: 2,000)$ secondary antibodies. The protein blots were visualized by enhanced chemiluminescence (Millipore, Billerica, MA, USA) with Chemilumino Analyzer LAS-3000 (Fujifilm, Tokyo, Japan). Quantity One 1-D Analysis software (Bio-Rad Laboratories) was used to analyze the band density of each blot. Anti $\beta$-actin was also used as an internal control and to confirm that each sample contained a similar quantity of protein.

Statistical analysis. All the experimental data presented were confirmed in at least three independent experiments, unless otherwise indicated. The experimental results are expressed as the mean \pm standard deviation. Collected data were statistically 
A

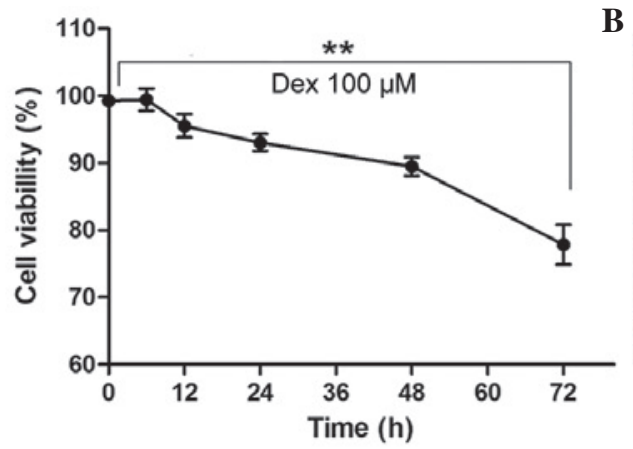

C

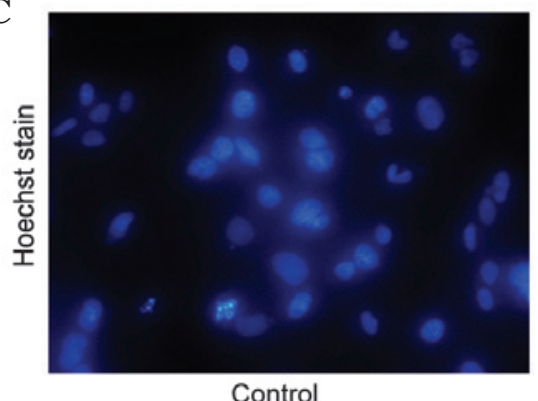

Control

$\mathbf{E}$

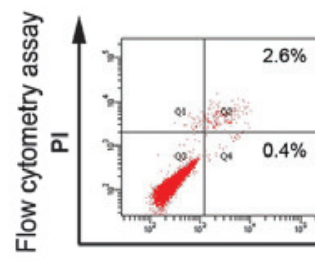

Dex $100 \mu \mathrm{M}$

$\mathrm{Oh}$

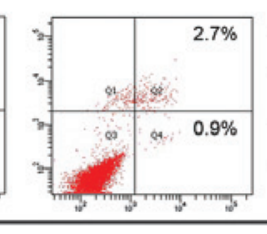

$6 \mathrm{~h}$

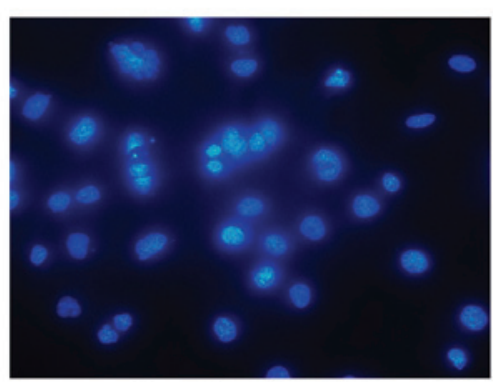

Dex $100 \mu \mathrm{M}$ for $72 \mathrm{~h}$

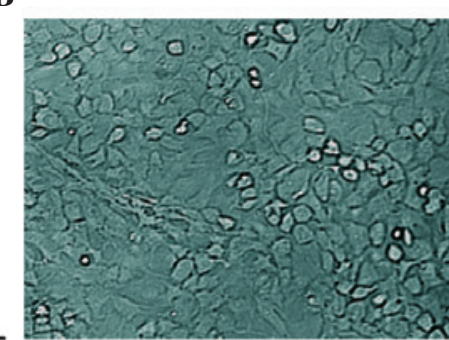

Control

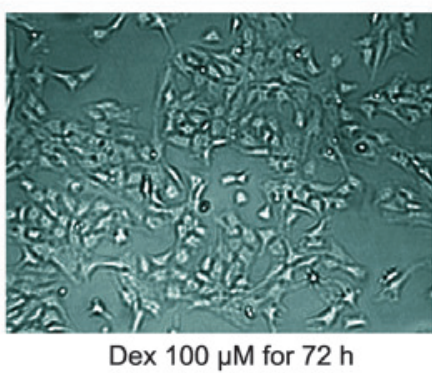

D

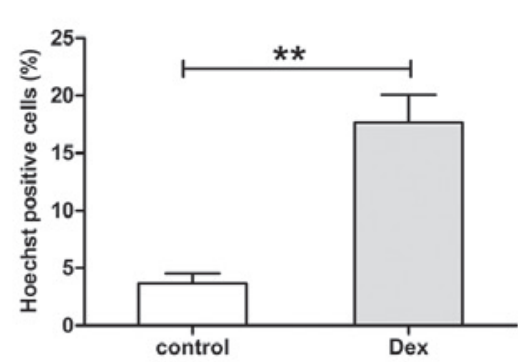

Figure 1. Inhibition rate and induction of apoptosis in human chondrocytes treated with Dex. (A) At different times of Dex treatment, the cell viability index $(\%)$ of six groups was assessed using the CCK- 8 assay. The results are expressed as the mean \pm SD and significant differences are indicated by asterisks ( $\left.{ }^{* *} \mathrm{P}<0.01\right)$. (B) Morphological changes were observed by inverted microscopy (magnification, x200). (C) Following Dex treatment for 72 h, apoptotic cells were stained with Hoechst 33342 and visualized by fluorescence microscopy (magnification, x400). (D) Hoechst-positive cells (bright blue with nuclear fragmentation) are expressed as the mean \pm SD and significant differences are indicated by asterisks $\left({ }^{* *} \mathrm{P}<0.01\right.$, vs. control). (E) Percentage of apoptotic cells following Dex treatment for different periods of time. The apoptotic index was determined by flow cytometry following Annexin V-FITC and PI staining. Dex, dexamethasone; FITC, fluorescein isothiocyanate; SD, standard deviation; PI, propidium iodide.

analyzed using a one-way analysis of variance and the Fisher's least-significant difference test with SPSS 19.0 software (SPSS Inc., Chicago, IL, USA). $\mathrm{P}<0.05$ was considered to indicate a statistically significant difference.

\section{Results}

Effect of GC treatment on the viability of human chondrocytes. It has been previously observed that, following $72 \mathrm{~h}$ exposure, Dex at $100 \mu \mathrm{M} / 1$ suppressed cell growth significantly (18). Therefore, in the present study, the short-term effect of Dex stimulation on the viability of chondrocytes was determined using the CCK- 8 assay. As shown in Fig. 1A and B, during short-term treatment (6-24 h), the average viability of chondrocytes remained $>90 \%$. However, following $72 \mathrm{~h}$ treatment, the viability of cells was decreased significantly $(\mathrm{P}<0.01)$ and cell morphological changes were observed. To further determine the cytotoxicity of Dex on chondrocytes, flow cytometry and Hoechst staining were used to assess apoptosis. As shown in Fig. 1C-E, following $100 \mu \mathrm{M} / 1$ Dex treatment for 6, 12, 24 and $48 \mathrm{~h}$, a slight increase in the rate of apoptosis was detected (3.6-9.2\%). However, following treatment for $72 \mathrm{~h}$, the rate of apoptosis increased to $16.7 \%$. Furthermore, Hoechst 33342 staining showed that cells treated with Dex for $72 \mathrm{~h}$ exhibited an increased ratio of Hoechst-labeled (bright blue nuclei indicative of apoptosis) cells compared with untreated cells $\left({ }^{* *} \mathrm{P}<0.01\right)$. These results indicated that short-term Dex treatment did not induce significant levels of apoptosis-associated cell death in chondrocytes; however, long-term treatment may induce apoptosis significantly.

Short-term GC treatment induces autophagy in chondrocytes. To investigate the autophagic activity following Dex treatment, MDC staining, TEM and western blot analysis were used. As shown in Fig. 2A and D, $100 \mu \mathrm{M} / 1$ Dex induced autophagy in a time-dependent manner. Between 6 and $24 \mathrm{~h}$ of treatment, MDC-labeled vacuoles were significantly increased. By contrast, following 48 and $72 \mathrm{~h}$ Dex treatment, a decrease in the occurrence of cell morphological changes and MDC-labeled vacuoles was observed. TEM scanning showed a large number of free membrane structures and double-membrane vacuoles, which contained portions of cytosol and organelles, in the cytoplasm following treatment 
A

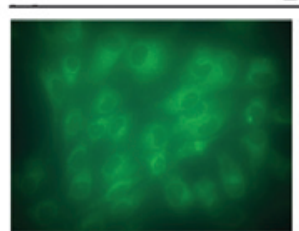

Control

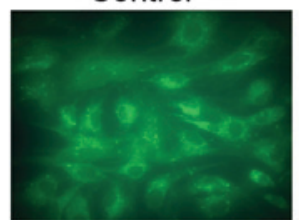

$24 \mathrm{~h}$

Dex $100 \mu \mathrm{M}$ (MDC stain)

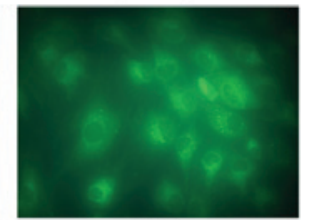

$6 \mathrm{~h}$

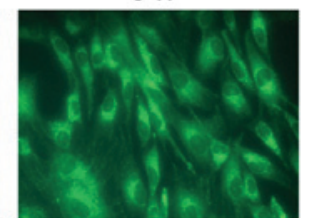

$48 \mathrm{~h}$

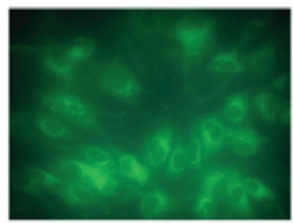

$12 \mathrm{~h}$

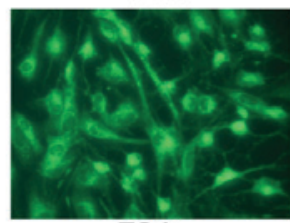

$72 \mathrm{~h}$

B

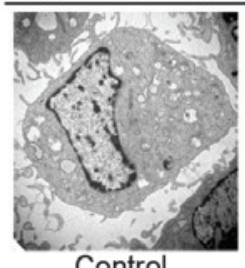

Dex $100 \mu \mathrm{M}$ (TEM analysis)

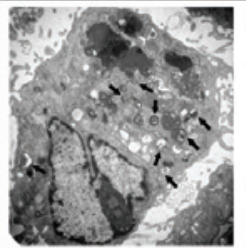

$24 \mathrm{~h}$

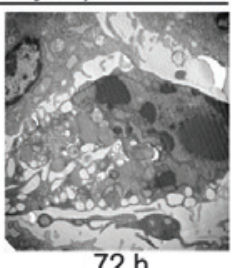

$72 \mathrm{~h}$

C

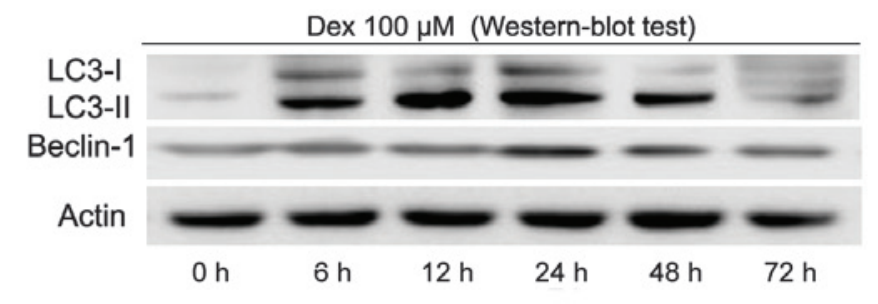

D
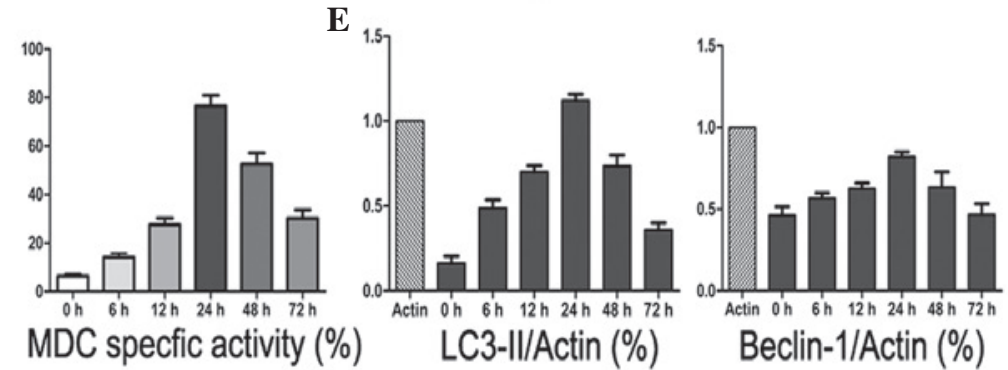

Figure 2. State of autophagy in human chondrocytes following Dex treatment. (A) MDC-labeled vacuoles were examined by fluorescence microscopy (magnification, x400). (B) Formation of autophagic vacuoles were observed by TEM (magnification, x15,000). Autophagic vacuoles are indicated by black arrows. (C) Western blot analysis of LC3-I, LC3-II and beclin-1 following incubation with Dex for different times. $\beta$-actin was used as a internal control. (D) The specific activity index (\%) was obtained by flow cytometry. (E) Levels of proteins were normalized with respect to the $\beta$-actin band density using Bio-Rad Quantity One software. Data are expressed as the mean \pm standard deviation. Dex, dexamethasone; MDC, monodansylcadaverine; TEM, transmission electron microscopy; LC3, microtubule-associated protein light chain 3.

with Dex for $24 \mathrm{~h}$ as compared with untreated cells. These structures resembled pre-autophagosomal structures (PAS) or autophagosomes (Fig. 2B, black arrows scale bars). Western blot analysis showed that LC3-II and Beclin-1 protein levels significantly increased between 6 and $24 \mathrm{~h}$ of treatment (Fig. 2C and E). However, this trend was reversed following long-term exposure to Dex. These observations suggested that short-term treatment with GCs may induce autophagy in chondrocytes.

Effects of activation of autophagy by Rapa on apoptosis of chondrocytes. It has been reported that Rapa may induce autophagy in chondrocytes. To further confirm the protective effect of Rapa in chondrocyte exposure to GC, the cells were pretreated with Rapa $2 \mathrm{~h}$ prior to treatment with Dex. The results suggested that, compared with Dex only treatment, pre-culture of the cells with Rapa enhanced the cell viability significantly $(\mathrm{P}<0.05$; Fig. $3 \mathrm{~A})$ and reduced the apoptotic rates (Fig. 3B). Furthermore, the activity of autophagy was stimulated by Rapa. The number of MDC-specific vacuoles was significantly increased ( $\mathrm{P}<0.01$; Fig. $3 \mathrm{C}$ and D). Finally, LC3-II protein expression was also upregulated, whereas the expression of apoptosis-associated gene caspase- 9 and the protein PARP were downregulated (Fig. 3E and F). These results suggested that the apoptosis induced by Dex may be through the activation of the caspase signaling pathway and that Rapa may maintain chondrocyte survival in the presence of GC by enhancing autophagy and suppressing apoptosis. 
A

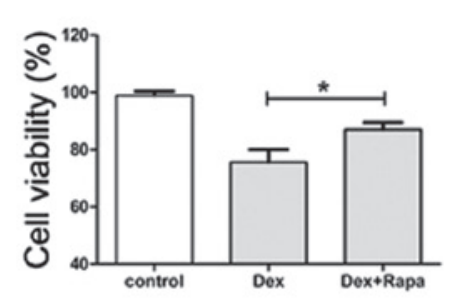

C

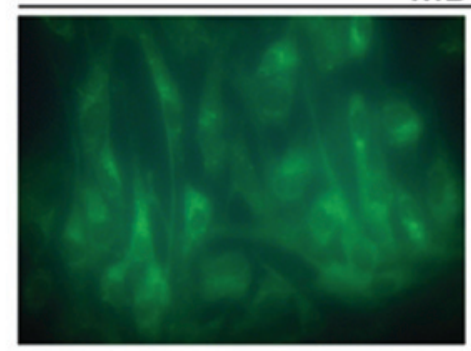

Dex

B

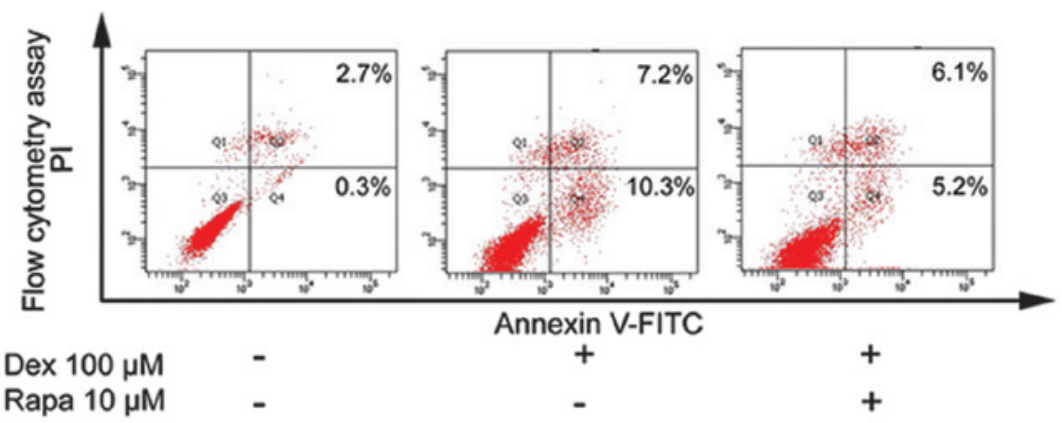

D

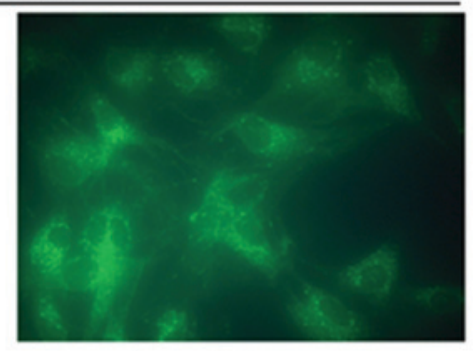

Dex+Rapa

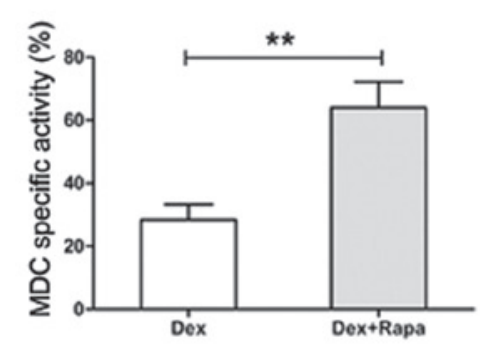

\section{E Cleaved caspase-9}

Cleaved PARP

LC3-I

LC3-II

Actin

Dex $100 \mu \mathrm{M}$

Rapa $10 \mu \mathrm{M}$

F

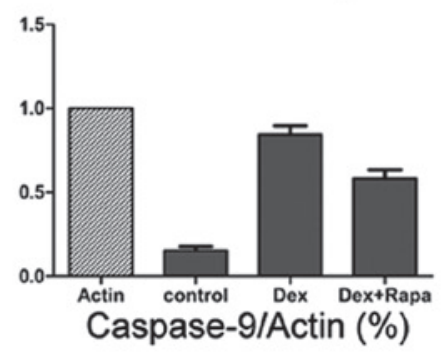

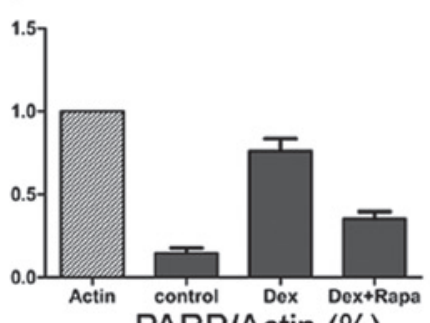

PARPIActin (\%)
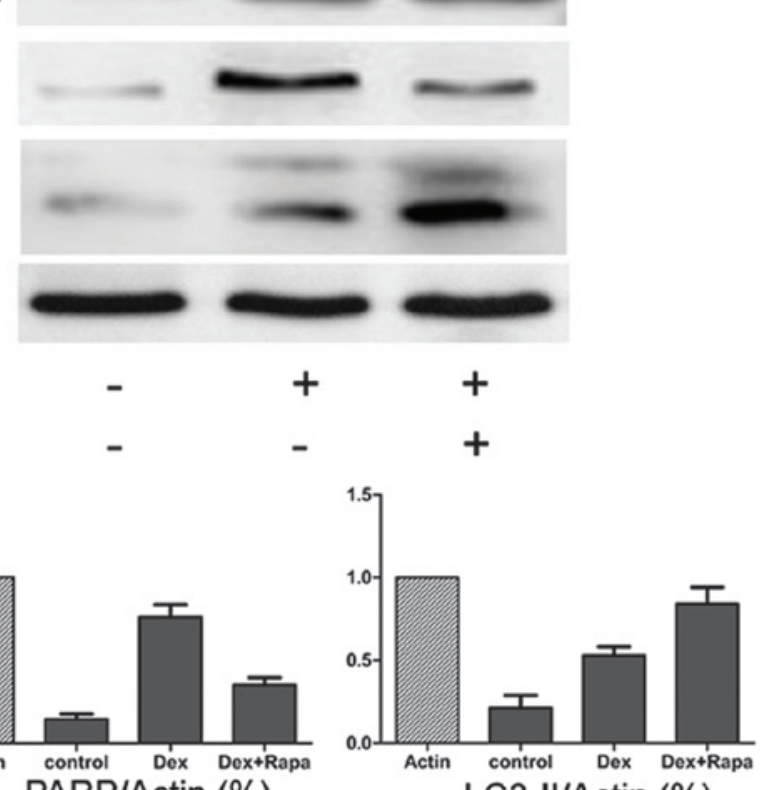

Figure 3. Effects of activation of autophagy by Rapa. (A) Chondrocytes were pretreated with or without Rapa and exposed to Dex for 72 h. The cell viability index (\%) was obtained using a CCK-8 assay. Data are expressed as the mean \pm SD and significant differences are indicated by asterisks $\left({ }^{*} \mathrm{P}<0.05\right.$, vs. Dex). (B) Following treatment, the apoptotic index was determined by flow cytometry following Annexin V-FITC and PI staining. (C) Following Dex only or pretreatment with Rapa with subsequent Dex exposure for $72 \mathrm{~h}$, the MDC-labeled vacuoles in chondrocytes were examined by fluorescence microscopy (magnification, $\mathrm{x} 400$ ). (D) The specific activity index (\%) was obtained by flow cytometry. Data are expressed as the mean \pm SD and significant differences are indicated by asterisks ( $\left({ }^{* *} \mathrm{P}<0.01\right.$, vs. Dex). (E) Western blot analysis for cleaved caspase-9, cleaved PARP, LC3-I and LC3-II protein following $72 \mathrm{~h}$ of incubation of untreated samples, Dex-treated samples and samples pretreated with Rapa followed by Dex treatment. $\beta$-actin was used as a internal control. (F) Levels of proteins were normalized to the $\beta$-actin band density using Bio-Rad Quantity One software. Data were expressed as the mean \pm SD. Rapa, rapamycin; Dex, dexamethasone; MDC, monodansylcadaverine; FITC, fluorescein isothiocyanate; SD, standard deviation; PI, propidium iodide; LC3, microtubule-associated protein light chain 3; PARP, poly (adenosine diphosphate-ribose) polymerase.

\section{Discussion}

$\mathrm{OA}$ is the most common type of joint disease worldwide, which is mainly characterized by chronic, irreversible degradation and erosion of cartilage $(1,22)$. For the majority of patients, joint pain and movement disorders are the primary clinical symptoms. At 
early stages, if the cartilage damage is not serious and joint pain is the major complaint, nonoperative therapy is preferred by the majority of clinicians (23). Glucocorticoid drugs, including Dex and prednisone, are effective anti-inflammatory agents in intra-articular injection therapy. Numerous studies have demonstrated the positive effects of GC in the treatment of early OA (24,25). However, GC treatment alters cartilage metabolism and changes the intra-articular environment $(7,8,26)$. Therefore, a number of studies have hypothesized that the repeated use of GC may facilitate cartilage degeneration $(27,28)$. In a recent study, long-term GC treatment was observed to inhibit the growth of chondrocytes and restrain the synthesis of the extracellular matrix (20). In the present study, it was observed that short-term GC treatment did not significantly reduce chondrocyte viability.

Autophagy, or the autophagic process, is a well-conserved mechanism among species and has been confirmed to be important in various biological events $(9,10,11,28)$. This system is characterized by the formation of double-membrane autophagic vesicles, which contain cytoplasm, and/or organelle degradation by lysosomes for material recycling and ATP generation (29). Among the human autophagy genes, Beclin-1 and LC3 are major regulators and markers of the autophagy pathway (30). Beclin-1 forms a complex with type III phosphatidylinositol that allows nucleation of the autophagic vesicles. During the process of autophagy activation, LC3-I is converted into LC3-II, which is then attached to the membrane of the autophagosome.

Chondrocytes are involved in maintaining a balance between synthesis and degradation of the ECM. Therefore, cell death is associated with the breakdown of cartilage (31-33). In 2004, the term 'chondroptosis' was the first to define this type cell death, which includes classical apoptosis and autophagy (34). In a number of instances, the cell switches between the above two responses in a mutually exclusive manner (35). As for the non-apoptotic mechanism, numerous studies have examined the roles of autophagy in normal cartilage metabolism and pathological conditions. Normal chondrocytes have a certain degree of autophagy and the aging-associated loss is linked with cell death (12). By contrast, the decreased autophagy with age may be an explanation for OA and activation of autophagy may reduce the severity of experimental OA (36). Therefore, in the early stages of OA, autophagy may be an adaptive response to avoid chondrocyte death (37). However, in deep zones of OA cartilage, due to the abnormal subchondral bone ossification, apoptosis is the only death process observed (37). In the present study, an increase in autophagic activity induced by short-term GC treatment was detected. The results show that only $6 \mathrm{~h}$ of chondrocyte exposure to Dex caused an upregulation in cell autophagy. This trend reached a maximum following $24 \mathrm{~h}$ of treatment. Continuous exposure to Dex led to the downregulation of autophagic activity. These resuls were obtained by MDC staining, which identified these trends in the autophagic activity of cells. The above results suggest that short-term treatment with GCs may also activate autophagy, whereas long-term exposure to GCs increases apoptosis. Autophagy is likely to be a self-protective process in chondrocytes in response to stimulation with GC.

To further determine the role of pharmacologically enhanced autophagy associated with the effects of Dex on chondrocyte viability, Rapa was employed as an autophagy inducer. The results suggested that, compared with Dex only treatment, Rapa enhances the viability of the cells after $72 \mathrm{~h}$. Next, western blot analysis confirmed that the pathological apoptosis markers caspase-9 and PARP were downregulated by Rapa, while the autophagy marker LC3 was upregulated. Rapa is a specific inhibitor of mTOR kinase, which regulates autophagy activation (18). mTOR is a nutrient-sensing kinase that integrates input from the energy status of the cell. Therefore, this kinase controls the ribosomal biogenesis and protein synthesis $(16,38)$. It has been demonstrated that this kinase is also involved in the cell death of chondrocytes in OA. Firstly, the activation of mTOR may suppress chondrocyte proliferation and differentiation by phosphorylation of the ribosomal protein S6 (39). Secondly, the inhibition of autophagy by mTOR caused OA-like changes in gene expression (40). Furthermore, mTOR inhibition may suppress the expression of A disintegrin and metalloproteinase with thrombospondin motifs 5 and interleukin (IL)-1 $\beta$ (36). Induction of proteinase expression in chondrocytes by IL-1 $\beta$ was reactive oxygen species (ROS)-dependent. Furthermore, increased ROS activity has been suggested to inhibit the growth factor expression and enhance the production of matrix metalloproteinases (41). However, the activation of autophagy by Rapa markedly reduces the ROS levels induced by IL-1 $\beta$ and reduces the severity of experimental OA $(35,36,42,43)$. GCs function as an anti-inflammatory agent. Therefore, in the present study, it was hypothesized that the apoptosis of chondrocytes may not be associated with the toxicity of inflammatory factors, but with the metabolic stress caused by GCs. Thus, pharmacologically enhanced autophagy by Rapa may act as an adaptive response to protect the chondrocytes from a relatively long-term exposure to GCs.

In conclusion, in regard to chondrocytes, the self-activation of autophagy is a protective mechanism against apoptosis under short-term treatment with GCs. However, persistent exposure to GCs may cause the cell to become dysfunctional, resulting in apoptosis. Furthermore, pharmacologically enhanced autophagy by Rapa provided novel insights into the treatment of the adverse effects of GCs on cartilage. Further study is required to elucidate the interaction between ECM synthesis and autophagy in chondrocytes.

\section{Acknowledgements}

The authors would like to thank Mr Wang Chunlei for technical assistance.

\section{References}

1. Felson DT and Zhang Y: An update on the epidemiology of knee and hip osteoarthritis with a view to prevention. Arthritis Rheum 41: 1343-1355, 1998.

2. Larsson E, Erlandsson Harris H, Larsson A, Månsson B Saxne T and Klareskog L: Corticosteroid treatment of experimental arthritis retards cartilage destruction as determined by histology and serum COMP. Rheumatology (Oxford) 43: 428-434, 2004.

3. Hollander JL: Intra-articular hydrocortisone in arthritis and allied conditions; a summary of two years' clinical experience. J Bone Joint Surg Am 35-A: 983-990, 1953.

4. Flanagan J, Casale FF, Thomas TL and Desai KB: Intra-articular injection for pain relief in patients awaiting hip replacement. Ann R Coll Surg Engl 70: 156-157, 1988. 
5. Friedman DM and Moore ME: The efficacy of intraarticular steroids in osteoarthritis: a double-blind study. J Rheumatol 7: $850-856,1980$

6. Lambert RG, Hutchings EJ, Grace MG, Jhangri GS, Conner-Spady B and Maksymowych WP: Steroid injection for osteoarthritis of the hip: a randomized, double-blind, placebo-controlled trial. Arthritis Rheum 56: 2278-2287, 2007.

7. Hainque B, Dominice J, Jaffray P, Ronot X and Adolphe M: Effects of dexamethasone on the growth of cultured rabbit articular chondrocytes: relation with the nuclear glucocorticoidrecepter complex. Ann Rheum Dis 46: 146-152, 1987.

8. Héraud F, Héraud A and Harmand MF: Apoptosis in normal and osteoarthritic human articular cartilage. Ann Rheum Dis 59: 959-965, 2000

9. Mizushima N, Levine B, Cuervo AM and Klionsky DJ: Autophagy fights disease through cellular self-digestion. Nature 451: 1069-1075, 2008 .

10. Levine B and Kroemer G: Autophagy in the pathogenesis of disease. Cell 132: 27-42, 2008

11. Martinet W, Agostinis P, Vanhoecke B, Dewaele M and De Meyer GR: Autophagy in disease: a double-edged sword with therapeutic potential. Clin Sci (Lond) 116: 697-712, 2009.

12. Caramés B, Taniguchi N, Otsuki S, Blanco FJ and Lotz M: Autophagy is a protective mechanism in normal cartilage, and its aging-related loss is linked with cell death and osteoarthritis. Arthritis Rheum 62: 791-801, 2010.

13. Caramés B, Kiosses WB, Akasaki Y, Brinson DC, Eap W, Koziol J and Lotz M: Glucosamine activates autophagy in vitro and in vivo. Arthritis Rheum 65: 1843-1852, 2013.

14. Shigemitsu K, Tsujishita Y, Hara K, Nanahoshi M, Avruch J and Yonezawa K: Regulation of translational effectors by amino acid and mammalian target of rapamycin signaling pathways. Possible involvement of autophagy in cultured hepatoma cells. J Biol Chem 274: 1058-1065, 1999.

15. Cardenas ME, Cutler NS, Lorenz MC, Di Como CJ and Heitman J: The TOR signaling cascade regulates gene expression in response to nutrients. Genes Dev 13: 3271-3279, 1999.

16. Dann SG, Selvaraj A and Thomas G: mTOR Complex1-S6K1 signaling: at the crossroads of obesity, diabetes and cancer Trends Mol Med 13: 252-259, 2007.

17. Harrison DE, Strong R, Sharp ZD, Nelson JF, Astle CM, Flurkey K, Nadon NL, Wilkinson JE, Frenkel K, Carter CS, Pahor M, Javors MA, Fernandez E and Miller RA: Rapamycin fed late in life extends lifespan in genetically heterogeneous mice. Nature 460: 392-395, 2009.

18. Pan T, Rawal P, Wu Y, Xie W, Jankovic J and Le W: Rapamycin protects against rotenone-induced apoptosis through autophagy induction. Neuroscience 164: 541-551, 2009.

19. Spilman P, Podlutskaya N, Hart MJ, Debnath J, Gorostiza O, Bredesen D, Richardson A, Strong R and Galvan V: Inhibition of mTOR by rapamycin abolishes cognitive deficits and reduces amyloid-beta levels in a mouse model of Alzheimer's disease. PLoS One 5: e9979, 2010.

20. Song YW, Zhang T and Wang WB: Gluococorticoid could influence extracellular matrix synthesis through Sox9 via p38 MAPK pathway. Rheumatol Int 32: 3669-3673, 2012.

21. Buss LW, Anderson C, Westerman E, Kritzberger C, Poudyal M, Moreno MA and Lakkis FG: Allorecognition triggers autophagy and subsequent necrosis in the cnidarian Hydractinia symbiolongicarpus. PLoS One 7: e48914, 2012.

22. Dobson F, Hinman RS, Roos EM, Abbott JH, Stratford P, Davis AM, Buchbinder R, Snyder-Mackler L, Henrotin Y Thumboo J, Hansen P and Bennell KL: OARSI recommended performance-based tests to assess physical function in people diagnosed with hip or knee osteoarthritis. Osteoarthritis Cartilage 21: 1042-1052, 2013.

23. Van Manen MD, Nace J and Mont MA: Management of primary knee osteoarthritis and indications for total knee arthroplasty for general practitioners. J Am Osteopath Assoc 112: 709-715, 2012.
24. Schumacher HR and Chen LX: Injectable corticosteroids in treatment of arthritis of the knee. Am J Med 118: 1208-1214, 2005.

25. Bjordal JM, Klovning A, Ljunggren AE and Slørdal L: Short-term efficacy of pharmacotherapeutic interventions in osteoarthritic knee pain: A meta-analysis of randomised placebo-controlled trials. Eur J Pain 11: 125-138, 2007.

26. Olney RC: Mechanisms of impaired growth: effect of steroids on bone and cartilage. Horm Res 72 (Suppl 1): 30-35, 2009.

27. Fubini SL, Todhunter RJ, Burton-Wurster N, Vernier-Singer M and MacLeod JN: Corticosteroids alter the differentiated phenotype of articular chondrocytes. J Orthop Res 19: 688-695, 2001.

28. Farkas B, Kvell K, Czömpöly T, Illés T and Bárdos T: Increased chondrocyte death after steroid and local anesthetic combination. Clin Orthop Relat Res 468: 3112-3120, 2010.

29. Gozuacik D and Kimchi A: Autophagy as a cell death and tumor suppressor mechanism. Oncogene 23: 2891-2906, 2004.

30. He C and Klionsky DJ: Regulation mechanisms and signaling pathways of autophagy. Annu Rev Genet 43: 67-93, 2009.

31. Lires-Deán M, Caramés B, Cillero-Pastor B, Galdo F, López-Armada MJ and Blanco FJ: Anti-apoptotic effect of transforming growth factor-beta1 on human articular chondrocytes: role of protein phosphatase 2A. Osteoarthritis Cartilage 16 1370-1378, 2008.

32. Blanco FJ, López-Armada MJ and Maneiro E: Mitochondrial dysfunction in osteoarthritis. Mitochondrion 4: 715-728, 2004.

33. Mobasheri A: Role of chondrocyte death and hypocellularity in ageing human articular cartilage and the pathogenesis of osteoarthritis. Med Hypotheses 58: 193-197, 2002.

34. Roach HI, Aigner T and Kouri JB: Chondroptosis: a variant of apoptotic cell death in chondrocytes? Apoptosis 9: 265-277, 2004.

35. Caramés B, Hasegawa A, Taniguchi N, Miyaki S, Blanco FJ and Lotz M: Autophagy activation by rapamycin reduces severity of experimental osteoarthritis. Ann Rheum Dis 71: 575-581, 2012

36. Sasaki H, Takayama K, Matsushita T, Ishida K, Kubo S, Matsumoto T, Fujita N, Oka S, Kurosaka M and Kuroda R: Autophagy modulates osteoarthritis-related gene expression in human chondrocytes Arthritis Rheum 64: 1920-1928, 2012.

37. Almonte-Becerril M, Navarro-Garcia F, Gonzalez-Robles A, Vega-Lopez MA, Lavalle C and Kouri JB: Cell death of chondrocytes is a combination between apoptosis and autophagy during the pathogenesis of Osteoarthritis within an experimental model. Apoptosis 15: 631-638, 2010

38. Kimball SR and Jefferson LS: Molecular mechanisms through which amino acids mediate signaling through the mammalian target of rapamycin. Curr Opin Clin Nutr Metab Care 7: 39-44, 2004.

39. Kim MS, Wu KY, Auyeung V, Chen Q, Gruppuso PA and Phornphutkul C: Leucine restriction inhibits chondrocyte proliferation and differentiation through mechanisms both dependent and independent of mTOR signaling. Am J Physiol Endocrinol Metab 296: E1374-E1382, 2009.

40. Chen J, Crawford R and Xiao Y: Vertical inhibition of the PI3K/Akt/mTOR pathway for the treatment of osteoarthritis. J Cell Biochem 114: 245-249, 2013.

41. Bohensky J, Terkhorn SP, Freeman TA, Adams CS, Garcia JA, Shapiro IM and Srinivas V: Regulation of autophagy in human and murine cartilage: hypoxia-inducible factor 2 suppresses chondrocyte autophagy. Arthritis Rheum 60: 1406-1415, 2009.

42. Lo YY, Conquer JA, Grinstein S and Cruz TF: Interleukin-1 beta induction of $\mathrm{c}$-fos and collagenase expression in articular chondrocytes: involvement of reactive oxygen species. J Cell Biochem 69: 19-29, 1998

43. Henrotin YE, Bruckner P and Pujol JP: The role of reactive oxygen species in homeostasis and degradation of cartilage. Osteoarthritis Cartilage 11: 747-755, 2003. 